\title{
Properties of LTP Induction in the CA3 Region of the Primate Hippocampus
}

Nathaniel N. Urban, Darrell A. Henze, David A. Lewis, and

German Barrionuevo ${ }^{1}$

Departments of Neuroscience and Psychiatry and

Center for the Neural Basis of Cognition

University of Pittsburgh

Pittsburgh, Pennsylvania 15260

\section{Abstract}

Activity-dependent changes in synaptic strength, such as long-term potentiation (LTP), have been proposed to underlie memory storage in the brains of all mammals, including humans. However, most forms of synaptic plasticity, including LTP, are studied almost exclusively in rodents and related species. Thus, the hypothesis that LTP is important in human memory relies on the assumption that LTP is similar in the primate and rodent brains. We have begun to test this hypothesis by studying the properties and mechanisms of LTP induction in area CA3 of hippocampal slices from cynomolgus monkeys. We have found that LTP can be induced reliably at both mossy fiber-CA3 and

collateral/associational-CA3 synapses in the primate brain, and that the properties of LTP induction at these synapses are similar to what we and others have observed in experiments using hippocampal slices from rodents. Also, we have investigated the role of opioids in mossy fiber synaptic transmission and LTP and have found no effect of the opioid antagonist naloxone nor the opioid agonist dynorphin on mossy fiber synaptic transmission or potentiation. These data suggest that LTP in the primate and rat brains has a similar induction mechanism and, thus, that the rodent is a useful animal model in which to study synaptic modification such as LTP.

\section{Introduction}

Much of the interest in long-term potentiation (LTP) stems from the hypothesis that long-lasting synaptic modification is required for the storage of certain types of memories. However, as has been noted by several investigators (McNaughton and Barnes 1990; Barnes 1995), the gap between our understanding of experimentally induced synaptic modification and associative memory is enormous. This gap grows to a chasm if it is specified that human memory is of greatest interest.

Since LTP was first described at excitatory synapses in the rabbit hippocampus (Bliss and Lomo 1973), many studies have investigated the mechanisms of induction, expression, and maintenance of LTP (for review, see Bliss and Collingridge 1993; Nicoll and Malenka 1995). Other studies have linked LTP to the storage of information in the mammalian brain (for review, see Eichenbaum and Otto 1993; Barnes 1995). Although much has been learned about the mechanisms and functional significance of LTP, data on LTP have been obtained almost exclusively from the rodent brain. [There is some controversy as to whether guinea pigs and rabbits, which are commonly used in LTP experiments, should be classsified as rodents (Graur et al. 1991; D'Erchia et al. 1996). For the purposes of this paper, we intend "rodent" to refer to (among others) rats, mice, rabbits, and guinea pigs.] Thus, the question of whether the study of LTP may some day shed light on the mechanisms underlying memory formation in humans, or even nonhuman primates, depends on the currently untested assumption that LTP has the same induction requirements in rodent and primate brains.

We have begun to test this assumption by examining the properties of LTP induction in area CA3 of hippocampal slices from healthy, young

\footnotetext{
${ }^{1}$ Corresponding author.
}

LEARNING \& MEMORY 3:86-95 @ 1996 by Cold Spring Harbor Laboratory Press ISSN1072-0502/96 \$5.00

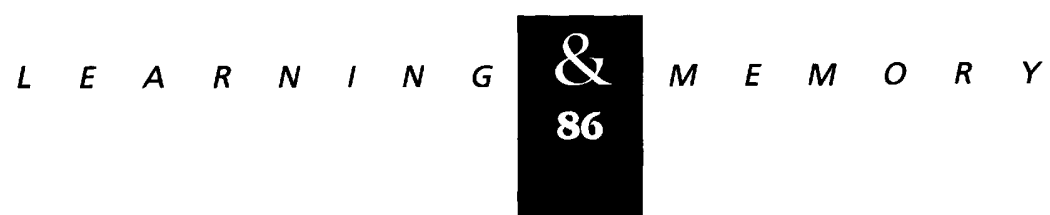


adult cynomolgus macaque monkeys. Specifically, we sought to (1) determine whether LTP can be induced at synapses in the primate hippocampus, (2) test whether LTP induction in primate area CA3 has the properties similar to the LTP we have studied in area CA3 of the rat (Urban and Barrionuevo 1996), and (3) investigate some properties of mossy fiber synaptic transmission and LTP induction for which species differences have been described between rats and guinea pigs (Salin et al. 1995; Williams and Johnston 1996).

\section{THREE FORMS OF LTP IN CA3}

LTP has been well studied at two excitatory synapses onto CA3 pyramidal neurons in rodents. LTP at synapses made by commissural/associational ( C/A) axons onto CA3 pyramidal neurons requires $N$-methyl-D-aspartate (NMDA) receptor activation (Harris and Cotman 1986; Zalutsky and Nicoll 1990) and is apparently indistinguishable from LTP at the synapse between Schaffer collaterals and CA1 pyramidal neurons (Zalutsky and Nicoll 1990; Hasselmo et al. 1995). In contrast, LTP at the synapse between mossy fiber axons and CA3 pyramidal neurons is independent of NMDAreceptor activation (Harris and Cotman 1986; Bradler and Barrionuevo 1990; Zalutsky and Nicoll 1990). The requirements for induction of mossy fiber LTP have been controversial. Some reports have concluded that induction of mossy fiber LTP requires postsynaptic calcium entry (Williams and Johnson 1989) and depolarization (Jaffe and Johnston 1990) and is therefore Hebbian (Hebb 1949; McNaughton and Morris 1987), whereas others have concluded that mossy fiber LTP is independent of postsynaptic activity (Zalutsky and Nicoll 1990; Katsuki et al. 1991; Castillo et al. 1994; Langdon et al. 1995) and is therefore nonHebbian.

These apparently contradictory results (cited above) can be explained by the hypothesis that both Hebbian and non-Hebbian mossy fiber LTP can be induced under different experimental conditions. Recently, we have reported data supporting this hypothesis (Urban and Barrionuevo 1996). In these experiments, which were performed in slices from Sprague-Dawley rats, brief bursts of high-frequency stimulation (B-HFS) induced a form of mossy fiber LTP that was blocked by preventing postsynaptic depolarization or calcium influx. In contrast, long trains of HFS induced a form of mossy fiber LTP that did not require postsynaptic depolarization or calcium influx. Based on these observations, we concluded that two distinct forms of NMDA-independent LTP can be induced at the mossy fiber-CA3 synapse in the rat hippocampus. One goal in the current study was to determine whether the three forms of LTP in rat CA3-NMDA-receptor dependent C/A LTP as well as Hebbian and non-Hebbian NMDA receptor independent mossy fiber LTP-have analogues in the primate.

\section{SPECIES-SPECIFIC MOSSY FIBER PHARMACOLOGY}

Studies of mossy fiber LTP are complicated by reports that mossy fiber synaptic transmission and LTP induction show different pharmacological sensitivities in rat and guinea pig. Lanthorn et al. (1984) reported that 50-100 $\mu \mathrm{M}$ DL 2-amino4-phosphorobutyric acid D/L AP4) suppressed mossy fiber synaptic transmission completely in the guinea pig, although it had no effect in the rat. Others have reported that dynorphin, as well as the $\mathrm{k}$-selective opioid agonist U69,593, selectively depresses mossy fiber synaptic transmission in slices from guinea pig (Williams and Johnston 1996), hamster, and mouse (Salin et al. 1995) but that these peptides are much less effective in slices from rats, especially of the Sprague-Dawley strain (Salin et al. 1995; Williams and Johnston 1996). The functional consequences of these pharmacological differences in mossy fiber synaptic transmission are unknown.

In addition to affecting basal synaptic transmission, opioid-receptor activation has been reported to play a role in the induction of mossy fiber LTP, although these reports are controversial. Following an early report that naloxone blocks induction of mossy fiber LTP in guinea pig slices (Martin 1983), other groups have reported similar observations in the rat, both in vivo (Derrick and Martinez 1994), as well as in vitro (Williams and Johnston 1996). However, Williams and Johnston (1996) did not observe an effect of naloxone on the induction of mossy fiber LTP in slices from guinea pig, thus failing to replicate the original observation of Martin in this species (1983). Finally, Salin et al. (1995) concluded that naloxone has no effect on the induction of mossy fiber LTP in either rat or guinea pig.

Although these data apparently are conflicting and thus difficult to interpret, they do indicate that 
Urban et al.

the effects of opioids on mossy fiber synaptic transmission and LTP may differ even in closely related species. Thus, it is difficult to determine whether either of these species will serve as a useful model of the role of opioids in mossy fiber LTP in human and nonhuman primates. Therefore, a second goal of these experiments was to study the effects of opioid agonists and antagonists on mossy fiber synaptic transmission and LTP in the primate.

\section{Materials and Methods}

\section{SPLICE PREPARATION}

Data were obtained from six young adult, male, cynomolgus monkeys (Macaca fascicularis). Tissue specimens from these animals were used in other investigations of cortical circuitry. All animals were treated according to the guidelines outlined in the NIH Guide to the Care and Use of Animals. Monkeys were initially anesthetized with ketamine $(25 \mathrm{mg} / \mathrm{kg}$, i.m. $)$, treated with dexamethazone $(0.5 \mathrm{mg} / \mathrm{kg}$, i.m. $)$ and atropine $(0.05 \mathrm{mg} / \mathrm{kg}$, s.c. $)$, and then intubated and maintained on $1 \%$ halothane in $28 \% \mathrm{O}_{2}$ /air during the surgical procedure. Animals were positioned in a stereotaxic apparatus, and a craniotomy was performed over the left prefrontal cortex as part of another study. Five minutes prior to the completion of this procedure, animals were given a second injection of ketamine. Animals were then anesthetized deeply with pentobarbital $(30 \mathrm{mg} /$ $\mathrm{kg}$, i.p.), removed from the stereotaxic apparatus, and ventilated mechanically with $28 \% \quad \mathrm{O}_{2}$. The chest was opened, the descending aorta clamped, and the animals perfused transcardially with cold modified artificial cerebrospinal fluid (ACSF) containing (concentrations in $\mathrm{mM}$ ) sucrose, 229.0; $\mathrm{KCl}, 1.9 ; \mathrm{Na}_{2} \mathrm{PO}_{4} \cdot 7 \mathrm{H}_{2} \mathrm{O}, 1.2 ; \mathrm{NaHCO}_{3}, 33.0$; dextrose, 10.0; $\mathrm{MgCl}_{2}, 10.0$; and kynurenic acid, 2.0; bubbled with $95 \% / 5 \% \mathrm{O}_{2} / \mathrm{CO}_{2}(\mathrm{pH} 7.4)$ (Aghajanian and Rasmussen 1989; Henze et al. 1996) at a flow rate of $270 \mathrm{ml} / \mathrm{min}$ for $4-5.5 \mathrm{~min}$. The brain was then rapidly removed (which took 5-6 min), and a $1-\mathrm{cm}$ thick coronal block containing the left hippocampus was removed and placed in cold modified ACSF. This coronal block was then trimmed and mounted to the stage of a vibratome and sliced into $400-500-\mu \mathrm{m}$ transverse sections (Fig. 1). Sections were then transferred into a holding chamber containing standard ACSF (concentrations in mM: $\mathrm{NaCl}, 125.0 ; \mathrm{KCl}, 2.0$; dextrose,

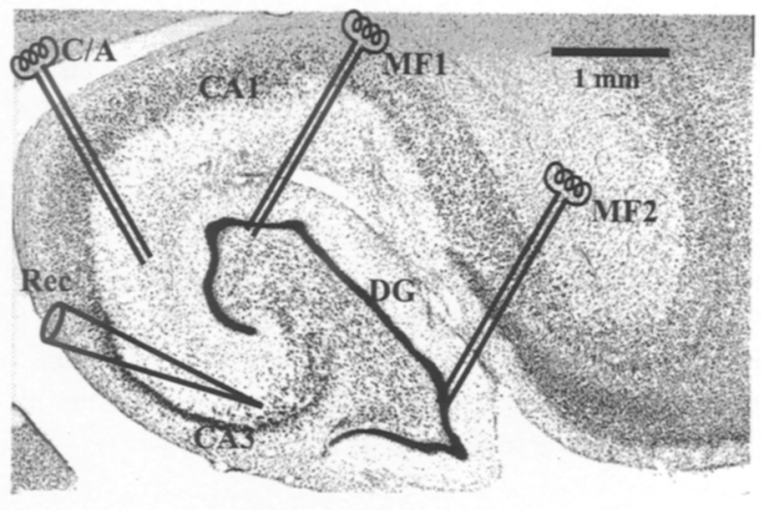

Figure 1: A section of the primate hippocampus showing electrode placement. A Nissl stained section coronal of the primate hippocampus showing usual positions of stimulating and recording electrodes in these experiments. (Rec) Extracellular recording pipette positioned in s. lucidum. In other experiments the recording electrode was placed in stratum radiatum. (C/A) Bipolar electrode placed in stratum radiatum used to stimulate collateral/associational fibers. (MF1/2) Bipolar electrodes placed in the dentate gyrus used to stimulate mossy fibers.

10.0; $\left.\mathrm{NaHCO}_{3}, 26.0\right)$ with high magnesium ( $6 \mathrm{~mm}$ $\mathrm{MgCl}_{2}$ ) and low calcium ( $1 \mathrm{~mm} \mathrm{CaCl}_{2}$ ) continuously bubbled with $95 \% \mathrm{O}_{2} / 5 \% \quad \mathrm{CO}_{2}$.

\section{STIMULATION AND RECORDING TECHNIQUES}

To maximize the yield and reliability of our experiments, and thus to reduce the number of slices and animals needed, all experiments were performed using extracellular recording techniques to measure population synaptic responses. Field EPSPs were recorded from slices submerged in standard ACSF containing $1.0 \mathrm{~mm}$ magnesium $\left(\mathrm{MgCl}_{2}\right)$ and $2.5 \mathrm{~mm}$ calcium $\left(\mathrm{CaCl}_{2}\right)$ at $32^{\circ} \mathrm{C}$. Extracellular glass electrodes $(1-3 \mathrm{M} \Omega$ ) were filled with $0.5 \mathrm{M} \mathrm{NaCl}$. Bipolar stimulating electrodes were made of nichrome wire. Test responses were evoked at $0.1 \mathrm{~Hz}$. In experiments in which two stimulating electrodes were used, pathways were considered to be independent if they showed no heterosynaptic paired pulse facilitation at a 40 msec inter-stimulus interval. All experiments report EPSP peak amplitude, which was well correlated with the initial slope of the EPSP in all cases in which both were monitored.

C/A synaptic responses in CA3 were evoked by placing the stimulating and recording electrodes in the stratum radiatum (see Fig. 1). In these recordings, electrode placement and stimu- 
lation intensity were adjusted to eliminate any evidence of an antidromically activated population spike. Responses evoked in this manner were indistinguishable from field EPSPs recorded in stratum radiatum of CA1 of these same slices in response to Schaffer collateral/commissural stimulation.

Mossy fiber responses were evoked using one or two stimulating electrodes placed in the granule cell layer of the dentate gyrus. In many slices, we confirmed that the slice angle used preserved the mossy fiber connection between the granule cells of the dentate gyrus and CA3 pyramidal cells by antidromically activating granule cells via a stimulating electrode placed in the stratum lucidum.

The complicated circuitry of area CA3 (Claiborne et al. 1993) makes isolation and identification of mossy fiber responses difficult. Thus, we used a set of criteria to distinguish mossy fiber field EPSPs from CA3 population spikes and from nonmossy fiber EPSPs elicited by activation of recurrent collaterals. This set of criteria was essentially the same as the criteria that we have used to identify mossy fiber field EPSPs in the rat (Castillo et al. 1994; Urban and Barrionuevo 1996). The criteria are: (1) The response must be recorded in stratum lucidum or, if stratum lucidum cannot be reliably identified in a given slice, in a region extending $\sim 100 \mu \mathrm{m}$ from the CA3 cell body layer. (2) The duration of the sink current must be $>4$ msec. Because we have observed that antidromically evoked population spikes in area CA3 last $\leqslant 2$ msec, this criterion allows us to distinguish mossy fiber EPSPs from CA3 population spikes. (3) A source rather than a sink current must be recorded in the stratum radiatum. This criterion allows us to distinguish mossy fiber from nonmossy fiber field EPSPs. Finally, electrode position and stimulation intensity were adjusted to minimize the positivity that sometimes follows the mossy fiber EPSP. This positivity may represent activation of collateral synapses and may complicate measurements of mossy fiber field EPSPs. In most experiments, the induction of NMDA receptor-independent LTP served to confirm that the responses recorded were, at least in part, mossy fiber in origin.

\section{INDUCTION AND MEASUREMENT OF LTP}

LTP was induced using one of two patterns of HFS (Urban and Barrionuevo 1996). Long trains of
HFS (L-HFS) consisted of 100 pulses at $100 \mathrm{~Hz}$ repeated 3 times at 10 -sec intervals. Brief trains of HFS (B-HFS) consisted of 8 pulses at $100 \mathrm{~Hz}$ repeated 10 times at 5 -sec intervals. The magnitude of LTP was determined by dividing the average amplitude of the responses obtained at $20 \mathrm{~min}$ after the end of the HFS by the average amplitude of the responses obtained in the 5 -min preceding the beginning of the HFS. All reports of LTP and all average time courses include all experiments in which the protocol (HFS plus any drugs) was given to a previously untetanized pathway. In experiments in which mossy fiber LTP was studied, $10 \mu \mathrm{M}$ MK-801 and $25 \mu \mathrm{M}$ D-APV were included in the bathing medium throughout the experiment to block NMDA-receptor dependent forms of LTP. When slices were not to be used for experiments examining NMDA-receptor dependent LTP, 2-10 $\mu \mathrm{M}$ MK-801 was added to the slice incubation medium as another precaution to ensure complete blockade of NMDA-receptor dependent forms of LTP.

\section{SOLUTION AND DRUGS}

All drugs were added to the bathing medium and allowed at least 20 min to reach effective concentration. Stock solutions of naloxone (10 mM) were made from the fresh powder and stored in the dark during the day of experiments. The APV concentration used in these experiments was effective at blocking NMDA-receptor-dependent LTP in rat CA1. The AP4 effectively blocked the field EPSPs evoked by lateral perforant path stimulation in the dentate gyrus in rat and monkey. Kynurenic acid (10 $\mathrm{mm}$ ) was dissolved directly into the recording medium and the $\mathrm{pH}$ of this solution was then adjusted to 7.4 using a $50 \%$ solution of $\mathrm{NaOH}$. Dynorphin stock solution ( $5 \mu \mathrm{M}$ ) was prepared daily from the fresh powder and kept at $\sim 4^{\circ} \mathrm{C}$ prior to being used.

All drugs were purchased from Sigma, with the exception of naloxone, APV, and MK-801 (RBI; Natick, MA). Waveforms shown are averages of 3-6 consecutive responses. All results are given as mean \pm S.E.M.

\section{Results}

LTP AT THE COLLATERAL/ASSOCIATIONAL (C/A) TO CA3 SYNAPSE

In five slices we tested whether the induction of LTP at the C/A synapse in the CA3 region re- 
quires activation of NMDA receptors. Stable field EPSPs were recorded in stratum radiatum in response to stimulation also in stratum radiatum. The addition of $25 \mu \mathrm{M}$ D-APV did not affect the amplitude of the field EPSP noticeably. After washing in the APV and recording more than $10 \mathrm{~min}$ of stable baseline, L-HFS (see Materials and Methods, 100 pulses at $100 \mathrm{~Hz}$ repeated 3 times) was applied. In the presence of APV, L-HFS resulted in no change in the amplitude of the field EPSP $(98 \pm 2 \%$ of control, $P>0.1, n=5$; Fig. $2 \mathrm{~A}$ ). In these same slices we then washed the APV for $\geqslant 20 \mathrm{~min}$ and administered L-HFS again, without adjusting the electrodes or the stimulation intensity. This second L-HFS resulted in significant LTP of the field EPSP ( $136 \pm 8 \%$ of baseline, $P<0.02, n=5$ ). We concluded that NMDA-dependent LTP can be induced at $\mathrm{C} / \mathrm{A}$ synapses in primate hippocampal area CA3.

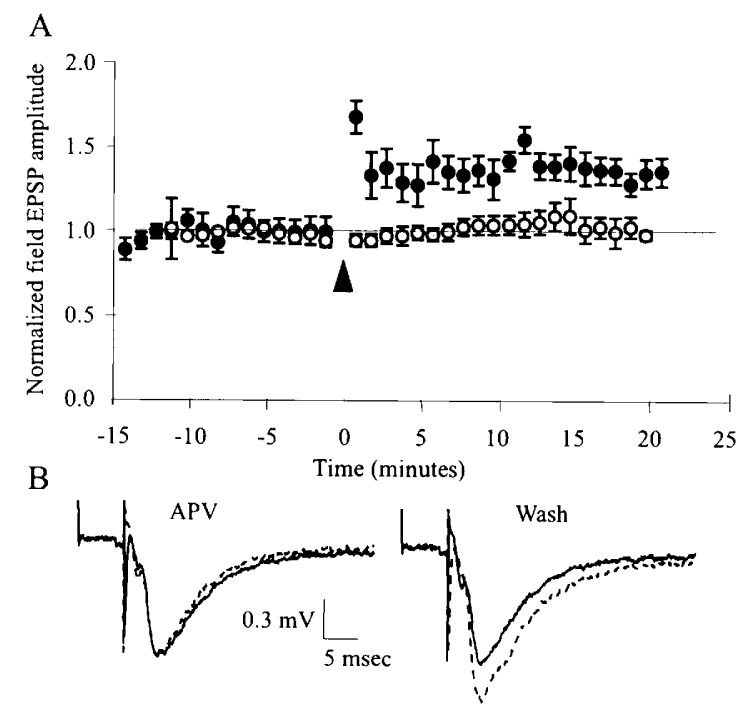

Figure 2: Demonstration of NMDA-receptor dependent LTP at the C/A to CA3 synapse. (A) Extracellular EPSPs were recorded in stratum radiatum of area $C A 3$ in response to stimulation of C/A fibers. EPSP peak amplitude plotted as a function of time. HFS (100 pulses at $100 \mathrm{~Hz}$ ) was applied at the time indicated by the triangle. In the presence of $25 \mu \mathrm{M}$ D-APV, HFS did not result in any potentiation of the field EPSP $(98 \pm 26 \%, n=4$; O). However, following washout of the APV, the identical HFS induced significant potentiation $(136 \pm 8 \%$, $n=4$; ). (B) Sample waveforms from a typical experiment. (Left) Waveforms recorded before (solid lines) and 20 min after (dashed lines) the HFS in APV. (Right) Waveforms recorded before (solid lines) and $20 \mathrm{~min}$ after (dashed lines) the HFS following the washout of APV.
SYNAPTIC TRANSMISSION AT THE MOSSY FIBER-CA3 SYNAPSE IN THE PRIMATE

In the guinea pig, but not in the rat, mossy fiber synaptic transmission can be blocked selectively by including either $50 \mu \mathrm{M}$ D/L-AP4 (Lanthorn et al. 1984) or $500 \mathrm{~nm}$ dynorphin (Salin et al. 1995; Weisskopf and Nicoll 1995) in the bathing medium. In guinea pig slices, complete synaptic blockade by AP 4 has been taken as evidence that dentate gyrus-evoked responses were uncontaminated by synaptic responses from $\mathrm{C} / \mathrm{A}$ synapses (Weisskopf and Nicoll 1995). We hoped to use this same strategy to identify mossy fiber responses in monkey slices. However, when the effect of AP4 on six putative mossy fiber responses was tested, a significant change in the amplitude of the field EPSP was never observed (98 $\pm 2 \%$ of baseline, $n=5$ ). Thus, we abandoned this strategy and based our identification of mossy fiber responses on criteria similar to those we had used to identify mossy fiber responses in the rat (see Materials and Methods).

In separate experiments, mossy fiber field EPSPs were unaffected by a 20-min exposure to 250 $1000 \mathrm{~nm}$ dynorphin (EPSP amplitude 104 $3 \%$ of baseline, $\boldsymbol{n}=5$ ). Following the washout of dynorphin, we bathed the slices in $25 \mu \mathrm{M}$ D-APV and 10 $\mu \mathrm{M}$ MK-801 for $20 \mathrm{~min}$. Application of HFS (either B-HFS or L-HFS) resulted in LTP, confirming that the responses being observed were mediated by mossy fiber synapses. In two of these same slices (as well as in four slices from rat hippocampus) dynorphin ( $500 \mathrm{nM}$ ) reduced the peak amplitude of a lateral perforant path evoked field EPSP recorded in the molecular layer of the dentate gyrus (reductions of $45 \%$ and $38 \%$ in the monkey slices). These results suggest that this lack of effect of dynorphin is specific to the primate mossy fiber synapse.

\section{INDUCTION OF MOSSY FIBER LTP IN THE PRIMATE}

INDUCTION OF TWO FORMS OF MOSSY FIBER LTP

Mossy fiber responses were recorded in the presence of $10 \mu \mathrm{M}$ MK-801 and $25 \mu \mathrm{M}$ D-APV. After recording stable baseline either B-HFS or L-HFS was applied. Both of these HFS protocols induced a potentiation of mossy fiber synaptic responses (B-HFS $=127 \pm 9 \%, P<0.05, n=6 ;$ L-HFS $=136$ $\pm 7 \%, P<0.05, n=7$; Fig. 3). In similar experi- 
ments performed in rat hippocampal slices, B-HFS of the dentate gyrus produced much less post-tetanic potentiation (PTP) of mossy fiber responses than did L-HFS (Urban and Barrionuevo 1996). This was not, however, the case in the experiments performed in slices from the monkey. In these experiments, both B-HFS and L-HFS resulted in potentiation that included a large, long-lasting PTP.

KYNURENIC ACID BLOCKS INDUCTION OF LTP BY BRIEF, BUT NOT LONG HFS

To test whether induction of mossy fiber LTP by these two patterns of HFS requires postsynaptic depolarization, we applied these patterns of HFS when synaptic transmission was blocked by $10 \mathrm{~mm}$ kynurenic acid. Our hypothesis was that kynurenic

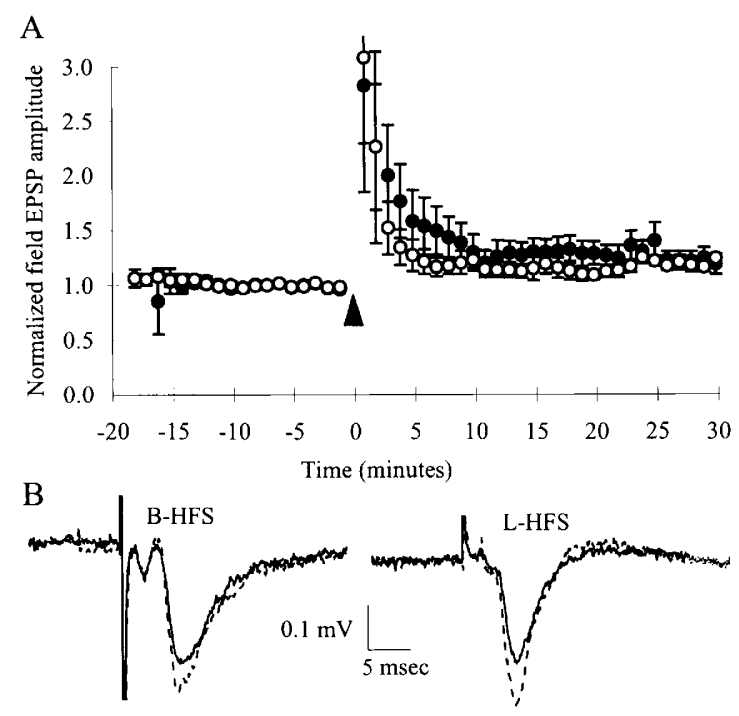

Figure 3: Demonstration of NMDA-receptor independent LTP at the mossy fiber-CA3 synapse. (A) Mossy fiber field EPSPs were recorded in stratum lucidum of area CA3 in response to stimulation of the dentate gyrus. EPSP peak amplitude is plotted as a function of time. Either L-HFS ( 100 pulses at $100 \mathrm{~Hz}$, repeated 3 times; ) or B-HFS (8 pulses at $100 \mathrm{~Hz}$, repeated 8 times; $\bigcirc$ ) was applied at the time indicated by the triangle. All experiments were performed in the presence of $25 \mu \mathrm{M}$ D-APV and $10 \mu \mathrm{M}$ MK-801 to block NMDA receptor-dependent forms of LTP. Both patterns of HFS resulted in similar LTP. (B) Sample waveforms from typical experiments. Waveforms were recorded before (solid lines) and 20 min after (dashed lines) the HFS. acid, by blocking AMPA receptor-mediated synaptic transmission, would prevent postsynaptic depolarization, and thus block the induction of Hebbian LTP (Ito and Sugiyama 1991; Castillo et al. 1994; Urban and Barrionuevo 1996).

One or two stimulating electrodes placed in the dentate gyrus were used to evoke mossy fiber field EPSPs. MK-801 (10 $\mu \mathrm{M})$ and D-APV ( $25 \mu \mathrm{M})$ were present throughout these experiments. After baseline responses were recorded, $10 \mathrm{~mm}$ kynurenic acid (KYN) was added to the recording medium. Synaptic responses were completely blocked $\sim 5 \mathrm{~min}$ after the addition of the KYN. After blockade was complete, we applied either B-HFS or L-HFS at the same stimulation intensity as was used for the test pulses. When two independent pathways were being stimulated in the same slice, the second pathway received no HFS. Immediately after the HFS was applied, the KYN was washed out and the field EPSPs were allowed to recover. In these experiments, LTP was assessed by comparing the amplitude of responses recorded 35 min following the washout of the KYN either (1) to the amplitude of responses recorded during the baseline period just prior to the addition of the KYN or (2) to the amplitude of responses recorded in the control, nontetanized pathway in the same slice. B-HFS, applied in the presence of $K Y N$, failed to induce a significant change in response amplitude $(110 \pm 12 \%$ of baseline before KYN, $P>0.1, n=5 ; 105 \pm 17 \%$ of control, nontetanized pathway, $P>0.1, n=3$; Fig. 4). LTP was, however, induced when these same slices received an identical B-HFS following the washout of the KYN ( $137 \pm 7 \%$ of control, $P<0.02$, $n=5$; Fig. 4 ). In contrast with B-HFS, L-HFS given in the presence of $\mathrm{KYN}$ resulted in a significant increase in EPSP amplitude following the washout of the KYN ( $137 \pm 11 \%$ of baseline, $P<0.02, n=6$; $142 \pm 14 \%$ of control, nontetanized pathway, $P<0.02, n=4$; Fig. 5).

INDUCTION OF MOSSY FIBER LTP BY BRIEF AND LONG HFS IS UNAFFECTED BY NALOXONE

Next, we investigated whether induction of LTP by either B-HFS or L-HFS requires activation of opioid receptors. Mossy fiber EPSPs were recorded in $10 \mu \mathrm{M}$ MK-801 and $25 \mu \mathrm{M}$ D-APV in response to dentate gyrus stimulation. After recording a stable baseline, naloxone (10-30 $\mu \mathrm{M})$

$$
\begin{array}{llllllllllllllll}
L & E & A & R & N & I & N & G & \bigotimes_{9} & M & E & M & O & R & Y
\end{array}
$$




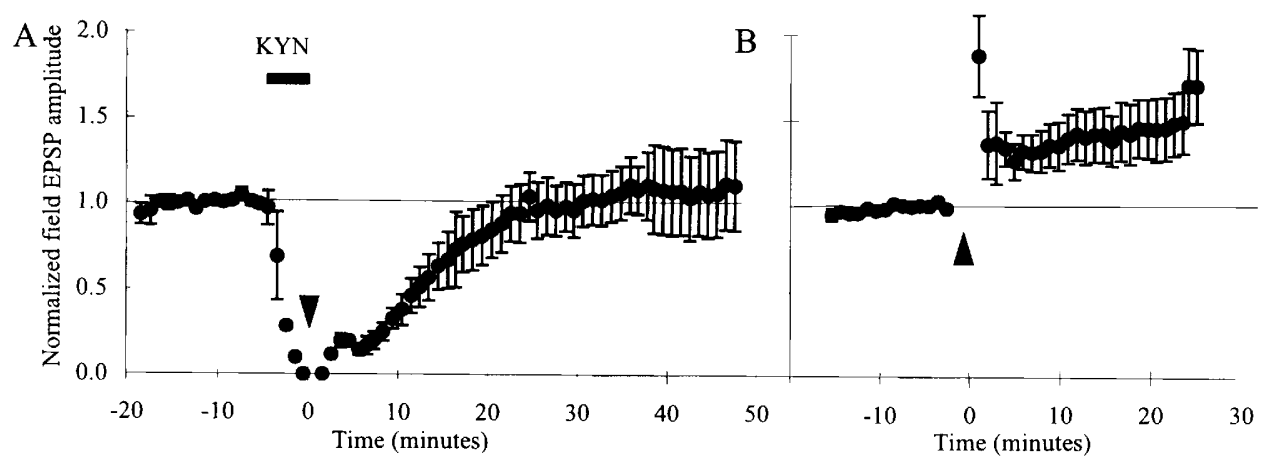

Figure 4: Induction of mossy fiber LTP by B-HFS requires glutamatergic ionotropic synaptic transmission. (A) After recording baseline field EPSPs, $10 \mathrm{~mm}$ kynurenic acid (KYN) was applied to block AMPA and NMDA receptors. When evoked EPSPs were eliminated, B-HFS was applied and the KYN was washed out. Thirty-five minutes after the washout of the KYN the response was not potentiated (response at $35 \mathrm{~min}$ post-HFS $=110 \pm 12 \%$ of baseline, $n=5$ ). An identical HFS applied following the washout of the KYN resulted in significant potentiation $(137 \pm 7 \%, n=4)$.

was added to the recording medium. The naloxone did not affect the amplitude of the mossy fiber field EPSP evoked by the test stimulation. After recording for $\geqslant 20 \mathrm{~min}$ in naloxone we applied either B-HFS or L-HFS. LTP was induced by both of these patterns of HFS (LTP by L-HFS: $130 \pm 5 \%$ of control, $P<0.02, n=8$; LTP by B-HFS $136 \pm 9 \%$, $P<0.02, n=9$; Fig. 6), and in both cases the time course of the potentiation was indistinguishable from the potentiation induced in control medium (Fig. 6). Based on these data, we conclude that in the primate hippocampus, induction of mossy fiber LTP does not require the activation of opioid receptors.

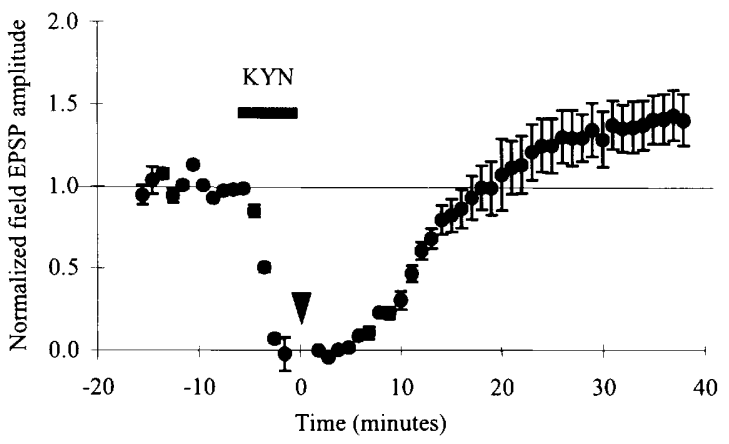

Figure 5: Induction of mossy fiber LTP by L-HFS does not require glutamatergic ionotropic synaptic transmission. After recording baseline field EPSPs, $10 \mathrm{~mm}$ kynurenic acid (KYN) was applied until evoked EPSPS were eliminated. B-HFS was then applied and the KYN was washed out. Thirty-five minutes after the washout of the KYN the response was significantly potentiated (response at 35 minutes post-HFS $=137 \pm 11 \%$ of baseline, $n=6)$.

\section{Discussion}

\section{SUMMARY}

This is the first full description of the properties of LTP induction in area CA3 of slices prepared from the healthy primate hippocampus. Our data suggest that the properties of LTP induction in the primate are similar to those that we have observed in the rat. Specifically, we report that (1) LTP can be induced at both $\mathrm{C} / \mathrm{A}$ and mossy fiber synapses onto pyramidal neurons in hippocampal area CA3; (2) LTP of the C/A synapse onto CA3 pyramidal cells requires activation of NMDA receptors whereas LTP of the mossy fiber synapse does not; (3) two forms of NMDA-receptor-independent LTP can be induced at the mossy fiber to CA3 synapse-one form requires post-synaptic depolarization and is therefore Hebbian, while the other form does not require postsynaptic depolarization and is therefore non-Hebbian; and (4) both of these forms of mossy fiber LTP can be induced in the presence of naloxone and thus neither form requires activation of opioid receptors for its induction. Also, we have demonstrated that mossy fiber synaptic transmission in this species of monkey is not blocked by bath application of AP4 or dynorphin.

\section{SPECIES DIFFERENCES}

The results reported here generally support the hypothesis that the rodent is a useful model for studying activity-dependent synaptic modifica- 


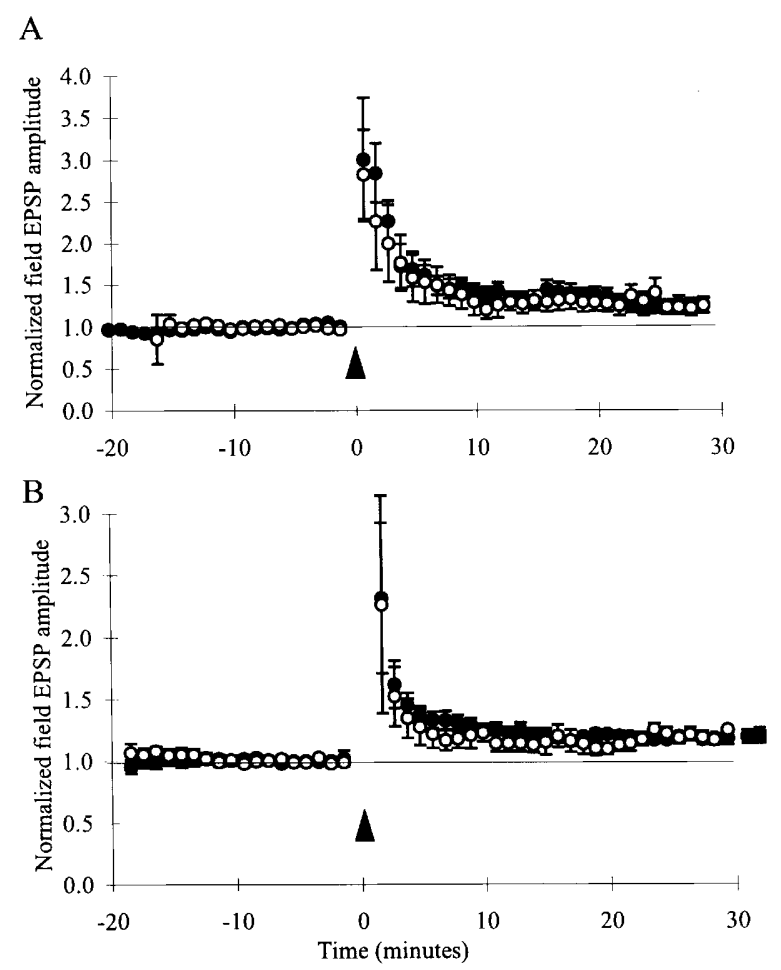

Figure 6: Naloxone does not affect the induction of mossy fiber LTP by B-HFS or L-HFS. Field EPSP peak amplitudes are plotted against time. The amplitude and time course of both mossy fiber LTP induced by $(A)$ L-HFS $(\mathrm{O}$; 0 , in Nalox) and (B) B-HFS (OO, in Nalox) are not significantly affected by the presence of $10-30$ $\mu \mathrm{M}$ naloxone in the bathing medium.

tions, such as LTP, that may underlie memory storage in primates. We have demonstrated that three different forms of rodent LTP have primate analogs and that these three forms of LTP have similar induction requirements in rat and primate. However, despite the qualitative similarity of LTP induction in primate and rat, direct comparison of primate versus rat mossy fiber LTP (Urban and Barrionuevo 1996) reveals that the magnitude of the potentiation observed in primate slices was significantly less than in rat slices. Although this difference in the magnitude of LTP could reflect a species difference, several other factors, including health of slices, age of animals, and environmental exposure, also may be responsible.

Because of unavoidable differences in slicing procedures, it is likely that the primate slices were not as healthy as slices obtained from rats using standard procedures. Despite our efforts (see Materials and Methods), the health of these primate slices may have been compromised by prolonged anoxia because the process of removing the brain from the cranial vault is more time-consuming in the primate than in the rat. Moreover, this procedure has a greater chance of resulting in mechanical damage to the hippocampus. If, as is commonly believed, LTP induction requires healthy synapses, then the smaller LTP magnitude in these experiments may have been mediated by slice health. Besides slice health, other differences that potentially could explain these results include differences in animal age and in the environment in which the animals were raised. Experiments in rodents suggest that age and exposure to an enriched environment may tend to saturate LTP, and thus these conditions may reduce the magnitude of LTP observed (Green and Greenough 1986; Moore et al. 1993). In light of these alternative explanations, we believe that the differences in the magnitude of LTP between rat and monkey probably reflect differences in slicing procedures and in the animals from which these slices were obtained, rather than a genuine species difference.

However, if the difference in the magnitude of LTP induction does reflect a real difference in synaptic plasticity in these species, then this could have important consequences for the simple model that predicts that more LTP should result in better memory. Such a model is called into question by behavioral data and theoretical analyses of neural networks. Several studies, in rat (for review, see Keith and Rudy 1990; Bannerman et al. 1995) and in monkey (Gutnikov and Gaffan 1996), have concluded that blockade of LTP may not be sufficient to prevent memory formation. Furthermore, work from neural network models demonstrates that the relationship between parameters governing the amount of plasticity that can be induced and performance on memory tasks is likely to be rather complicated. In some neural network models, the storage capacity (Treves and Rolls 1991) or pattern separation performance (O'Reilly and McClelland 1994) of a network is unaffected or even compromised by increasing the parameters (such as learning rate) that govern the amount of synaptic change that can occur. However, increasing such parameters may improve the performance of some networks on tasks involving small training sets. Thus, both experimental and theoretical studies suggest that the relationship between amount of synaptic plasticity and memory performance is unlikely to be straightforward, making the interpretation of a species difference in the magnitude of potentiation difficult.

$$
\begin{array}{llllllllllllllll}
\hline & E & A & R & N & I & N & G & \begin{array}{l}
\mathbf{X} \\
93
\end{array} & M & E & M & O & R & Y
\end{array}
$$


In addition to comparing primate and rodent LTP, in these experiments we also addressed two other issues related to possible species differences between primates and rodents. First, we observed that in the primate as in the rat, but unlike in the guinea pig, mossy fiber synaptic responses were unaffected by the application of $50 \mu \mathrm{M}$ D-AP4 or by $500 \mathrm{~nm}$ dynorphin. Second, we observed that naloxone $(10-30 \mu \mathrm{M})$ failed to block the induction of mossy fiber LTP by either B-HFS or L-HFS.

By necessity, neuroscientists rely heavily on animal models, even though their eventual goal is the understanding of some aspect of the human nervous system. Data acquired through the use of animal models have contributed to our knowledge of the mechanisms underlying function and dysfunction of the human nervous system. But the successful use of an animal model depends on empirical testing to determine whether key features of the model system are preserved across species. Such testing helps to determine whether our understanding of the model system contributes to our understanding of the human nervous system.

The experiments above lead us to conclude that the key features of three different forms of LTP are the essentially the same in primate as in rat. These results suggest that the induction requirements for these three forms of plasticity are conserved across most mammilian species, including humans, and thus that the data on LTP in the rat will be useful in our understanding of the processes of synaptic modification in humans. By testing this one critical hypothesis we hope to have slightly closed the chasm that stands between our understanding of synaptic plasticity and associative memory.

\section{Acknowledgments}

This work was supported by U.S. Public Health Service grants MH00519, MH 51234, and NS2428; a Howard Hughes Predoctoral fellowship to N.N.U., and a National Institute of Mental Health predoctoral fellowship $\mathrm{MH} 10474$ to D.A.H.

The publication costs of this article were defrayed in part by payment of page charges. This article must therefore be hereby marked "advertisement" in accordance with 18 USC section 1734 solely to indicate this fact.

\section{References}

Aghajanian, G.K. and K. Rasmussen. 1989. Intracellular studies in the facial nucleus illustrating a simple new method for obtaining viable motoneurons in adult rat brain slices. Synapse 3: 331-338.
Bannerman, D.M., M.A. Good, S.P. Butcher, M. Ramsay, and R.G.M. Morris. 1995. Distinct components of spatial learning revealed by prior training and NMDA receptor blockade. Nature 378: 182-186.

Barnes, C.A. 1995. Involvement of LTP in memory: Are we "Searching under the street light"? Neuron 15: 751-754.

Bliss, T.V.P. and G.L. Collingridge. 1993. A synaptic model of memory: Long-term potentiation in the hippocampus. Nature 361: 31-39.

Bliss, T.V.P. and T. Lomo. 1973. Long-lasting potentiation of synaptic transmission in the dentate area of the anaesthetized rabbit following stimulation of the perforant path. J. Physiol. 232: 331-356.

Bradler, J.E. and G. Barrionuevo. 1990. Heterosynaptic correlates of long-term potentiation induction in hippocampal CA3 neurons. Neuroscience 35: 265-271.

Castillo, P.E., M.G. Weisskopf, and R.A. Nicoll. 1994. The role of calcium channels in hippocampal mossy fiber synaptic transmission and long-term potentiation. Neuron 12: $261-269$.

Claiborne, B.J., Z. Xiang, and T.H. Brown. 1993. Hippocampal circuitry complicates analysis of long-term potentiation in mossy fiber synapses. Hippocampus 3: 115-122.

D'Erchia, A.M., C. Gissi, G. Pesole, C. Saccone, and U. Arnason. 1996. The guinea pig is not a rodent. Nature 381: 597-600.

Derrick, B.E. and J.L. Martinez, Jr. 1994.

Frequency-dependent associative long-term potentiation at the hippocampal mossy fiber-CA3 synapse. Proc. Natl. Acad. Sci. 91: 10290-10294

Eichenbaum, $\mathrm{H}$. and T. Otto. 1993. LTP and memory: Can we enhance the connection? Trends Neurosci. 16: 163-164.

Graur, D., W.A. Hide, and W.-H. Li. 1991. Is the guinea pig a rodent? Nature 351: 649-652.

Green, E.J. and W.T. Greenough. 1986. Altered synaptic transmission in dentate gyrus of rats reared in complex environments: Evidence from hippocampal slices maintained in vitro. I. Neurophysiol. 55: 739-750.

Gutnikov, S.A. and D. Gaffan. 1996. Systemic NMDA receptor antagonist CGP-40116 does not impair memory acquisition but protects againt NMDA neurotoxicity in rhesus monkeys. J. Neurosci. 16: 4041-405.

Harris, E.W. and C.W. Cotman. 1986. Long-term potentiation of guinea pig mossy fiber responses is not blocked by $\mathrm{N}$-methyl $\mathrm{D}$-aspartate antagonists. Neurosci. Lett. 70: 132-137.

Hasselmo, M.E., E. Schnell, and E. Barkai. 1995. Dynamics of learning and recall at excitatory recurrent synapses and 
cholinergic modulation in rat hippocampal region CA3. I. Neurosci. 15: 5249-5262.

Hebb, D.O. 1949. The organization of behavior. Wiley, New York, NY.

Henze, D.A., W.E. Cameron, and G. Barrionuevo. 1996. Dendritic morphology and its effects on the amplitude and rise time of synaptic signals in hippocampal CA3 pyramidal cells. J. Comp. Neurol. 69: 331-344.

Ito, I. and H. Sugiyama. 1991. Roles of glutamate receptors in long-term potentiation at hippocampal mossy fiber synapses. Neuroreport 2: 333-336.

Jaffe, D. and D. Johnston. 1990. Induction of long-term potentiation at hippocampal mossy-fiber synapses follows a Hebbian rule. I. Neurophys. 64: 948-960.

Katsuki, H., S. Kaneko, A. Tajima, and M. Satoh. 1991. Separate mechanisms of long-term potentiation in two input systems to CA3 pyramidal neurons of rat hippocampal slices as revealed by the whole-cell patch-clamp technique.

Neurosci. Res. 12: 393-402.

Keith, J.R. and J.W. Rudy. 1990. Why

NMDA-receptor-dependent long-term potentiation may not be a mechanism for memory and learning: Reappraisal of the NMDA-receptor-blockade strategy. Psychobiology

18: 251-257.

Langdon, R.B., J.W. Johnson, and G. Barrionuevo. 1995. Posttetanic potentiation and presynaptically induced long-term potentiation at the mossy fiber synapse in the rat hippocampus. J. Neurobiol. 26: 370-385.

Lanthorn, T.H., A.H. Ganong, and C.W. Cotman. 1984. 2-Amino-4-phosphonobutyrate selectively blocks mossy fiber-CA3 responses in guinea pig but not rat hippocampus. Brain Res. 290: 174-178.

McNaughton, B.L. and C.A. Barnes. 1990. From cooperative synaptic enhancement to associative memory: Bridging the abyss. Semin. Neurosci. 2: 403-416.

McNaughton, B.L. and R.G.M. Morris. 1987. Hippocampal synaptic enhancement and information storage within a distributed memory framework. Trends Neurosci.

10: $408-415$.

Martin, M.R. 1983. Naloxone and long-term potentiation of hippocampal CA3 field potentials in vitro. Neuropeptides 4: $45-50$.

Moore, C.L., M.D. Browning, and G.M. Rose. 1993. Hippocampal plasticity induced by primed burst, but not long-term potentiation, stimulation is impaired in area CA1 of aged Fischer 344. Hippocampus 3: 57-66.

Nicoll, R.A. and R.C. Malenka. 1995. Contrasting properties of two forms of long-term potentiation in the hippocampus. Nature 377: 115-118.
O'Reilly, R.C. and J.L. McClelland. 1994. Hippocampal conjunctive encoding, storage and recall: Avoiding a tradeoff. Hippocampus 4: 661-682.

Salin, P.A., M.G. Weisskopf, and R.A. Nicoll. 1995. A comparison of the role of dynorphin in the hippocampal mossy fiber pathway in guinea pig and rat. J. Neurosci. 15: 6939-6945.

Treves, A. and E.T. Rolls. 1991. What determines the capacity of autoassociative memories in the brain? Network 2: $371-397$.

Urban, N.N. and G. Barrionuevo. 1996. Induction of Hebbian and non-Hebbian LTP at the hippocampal mossy fiber synapse by distinct patterns of high frequency stimulation. J. Neurosci. 16: 4293-4299.

Weisskopf, M.G. and R.A. Nicoll. 1995. Presynaptic changes during mossy fiber LTP revealed by NMDA receptor-mediated synaptic responses. Nature 376: 256-259.

Williams, S. and D. Johnson. 1989. Long-term potentiation of hippocampal mossy fiber synapses is blocked by postsynaptic injection of calcium chelators. Neuron 3: 583-588.

Williams, S.H. and D. Johnston. 1996. Actions of endogenous opioids on NMDA receptor-independent long-term potentiation in area CA3 of the hippocampus. /. Neurosci. 16: 3652-3660.

Zalutsky, R.A. and R.A. Nicoll. 1990. Comparison of two forms of long-term potentiation in single hippocampal neurons. Science 248: 1619-1624.

Received June 14, 1996; accepted in revised form July 23, 1996. 


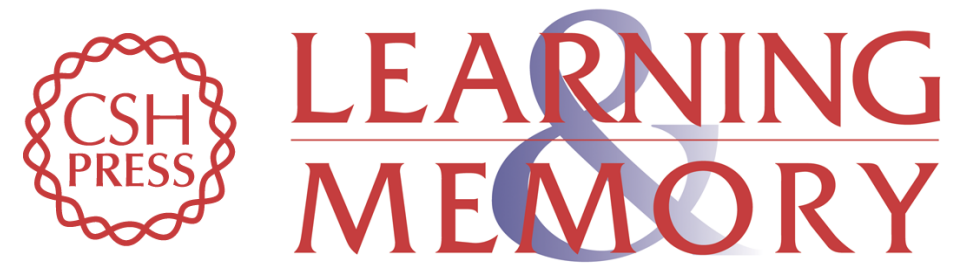

\section{Properties of LTP induction in the CA3 region of the primate hippocampus.}

N N Urban, D A Henze, D A Lewis, et al.

Learn. Mem. 1996, 3:

References This article cites 36 articles, 7 of which can be accessed free at: http://learnmem.cshlp.org/content/3/2-3/86.full.html\#ref-list-1

License

Email Alerting

Receive free email alerts when new articles cite this article - sign up in the box at the Service top right corner of the article or click here. 